\title{
Global existence and uniform decay for the one-dimensional model of thermodiffusion with second sound
}

\author{
Ming Zhang ${ }^{1 *}$ and Yuming Qin²
}

\section{"Correspondence: \\ zhangming87@hotmail.com \\ ${ }^{1}$ College of Information Science and \\ Technology, Donghua University, \\ Shanghai, 201620, P.R. China \\ Full list of author information is \\ available at the end of the article}

\begin{abstract}
In this paper, we investigate an initial boundary value problem for the one-dimensional linear model of thermodiffusion with second sound in a bounded region. Using the semigroup approach, boundary control and the multiplier method, we obtain the existence of global solutions and the uniform decay estimates for the energy.

MSC: 35B40; 35M13; 35Q79
\end{abstract}

Keywords: thermodiffusion; second sound; global existence; exponential decay

\section{Introduction}

In this paper, we investigate the global existence and uniform decay rate of the energy for solutions for the one-dimensional model of thermodiffusion with second sound:

$$
\begin{aligned}
& \rho u_{t t}-(\lambda+2 \mu) u_{x x}+\gamma_{1} \theta_{1 x}+\gamma_{2} \theta_{2 x}=0 \quad \text { in }(0,1) \times(0,+\infty), \\
& c \theta_{1 t}+\sqrt{k} q_{1 x}+\gamma_{1} u_{t x}+d \theta_{2 t}=0 \quad \text { in }(0,1) \times(0,+\infty), \\
& n \theta_{2 t}+\sqrt{D} q_{2 x}+\gamma_{2} u_{t x}+d \theta_{1 t}=0 \quad \text { in }(0,1) \times(0,+\infty), \\
& \tau_{1} q_{1 t}+q_{1}+\sqrt{k} \theta_{1 x}=0 \quad \text { in }(0,1) \times(0,+\infty), \\
& \tau_{2} q_{2 t}+q_{2}+\sqrt{D} \theta_{2 x}=0 \quad \text { in }(0,1) \times(0,+\infty),
\end{aligned}
$$

together with the initial conditions

$$
\begin{cases}u(x, 0)=u_{0}(x), & u_{t}(x, 0)=u_{1}(x), \\ \theta_{1}(x, 0)=\theta_{1}^{0}(x), & \theta_{2}(x, 0)=\theta_{2}^{0}(x), \\ q_{1}(x, 0)=q_{1}^{0}(x), & q_{2}(x, 0)=q_{2}^{0}(x),\end{cases}
$$

and the boundary conditions

$$
\left.u(x, t)\right|_{x=0,1}=\left.\theta_{1}(x, t)\right|_{x=0,1}=\left.\theta_{2}(x, t)\right|_{x=0,1}=0,
$$

where $u, \theta_{1}$, and $q_{1}$ are the displacement, temperature, and heat flux, $\theta_{2}$, and $q_{2}$ are the chemical potentials and the associated flux. The boundary conditions (1.7) model a rigidly

○2013 Zhang and Qin; licensee Springer. This is an Open Access article distributed under the terms of the Creative Commons Attribution License (http://creativecommons.org/licenses/by/2.0), which permits unrestricted use, distribution, and reproduction in any medium, provided the original work is properly cited. 
clamped medium with temperature and chemical potentials held constant on the boundary.

Here, we denote by $\lambda$, $\mu$ the material constants, $\rho$ the density, $\gamma_{1}, \gamma_{2}$ the coefficients of thermal and diffusion dilatation, $k, D$ the coefficients of thermal conductivity, $n, c, d$ the coefficients of thermodiffusion, and $\tau_{1}, \tau_{2}$ the (in general very small) relaxation time. All the coefficients above are positive constants and satisfy the condition

$$
n c-d^{2}>0
$$

The classical thermodiffusion equations were first given by Nowacki [1, 2] in 1971. The equations describe the process of thermodiffusion in a solid body (see, e.g., [1-5]):

$$
\left\{\begin{array}{l}
\rho u_{t t}-(\lambda+2 \mu) u_{x x}+\gamma_{1} \theta_{1 x}+\gamma_{2} \theta_{2 x}=0, \\
c \theta_{1 t}-k \theta_{1 x x}+\gamma_{1} u_{t x}+d \theta_{2 t}=0, \\
n \theta_{2 t}-D \theta_{2 x x}+\gamma_{2} u_{t x}+d \theta_{1 t}=0 .
\end{array}\right.
$$

There are many results about the classical thermodiffusion equations. By the method of integral transformations and integral equations, Nowacki [2], Podstrigach [6] and Fichera [7] investigated the initial boundary value problem for the linear homogeneous system. Gawinecki [8] proved the existence, uniqueness and regularity of solutions to an initial boundary value problem for the linear system of thermodiffusion in a solid body. Szymaniec [5] proved the $L^{p}-L^{q}$ time decay estimates along the conjugate line for the solutions of the linear thermodiffusion system. Using the results from [5], Szymaniec [9] obtained the global existence and uniqueness of small data solutions to the Cauchy problem of nonlinear thermodiffusion equations in a solid body. Using the semigroup approach and the multiplier method, Qin et al. [4] obtained the global existence and exponential stability of solutions for homogeneous, nonhomogeneous and semilinear thermodiffusion equations subject to various boundary conditions. Liu and Reissig [3] studied the Cauchy problem for one-dimensional models of thermodiffusion and explained qualitative properties of solutions and showed which part of the model has a dominant influence on wellposedness, propagation of singularities, $L^{p}-L^{q}$ decay estimates on the conjugate line and the diffusion phenomenon.

If we neglect the diffusion in (1.9), then we obtain the classical thermo-elasticity equations. Today models of type I (classical model of thermo-elasticity), of type II (thermal wave), of type III (visco-elastic damping) or second sound present some classification of models of thermo-elasticity (see, e.g., $[3,10,11]$ ). By considerations of the total energy equation and comparisons with the models of classical thermo-elasticity and thermodiffusion, we shall propose the linear one-dimensional model of thermodiffusion with second sound as mentioned above. Due to our knowledge, there exist no results for thermodiffusion models with second sound.

Our paper is organized as follows. In Section 2, we present some notations and the main result. Section 3 is devoted to the proof of the main result. 


\section{Notations and main result}

Let $\Omega=(0,1)$ and

$$
\begin{aligned}
& L_{*}^{2}(\Omega)=\left\{y \in L^{2}(\Omega) \mid \int_{0}^{1} y(x) d x=0\right\}, \\
& H_{*}^{1}(\Omega)=\left\{y \in H^{1}(\Omega) \mid \int_{0}^{1} y(x) d x=0\right\} .
\end{aligned}
$$

The associated first-order and second-order energy is defined by

$$
\begin{aligned}
E_{1}(t) & \equiv E_{1}\left(t ; u_{x}, u_{t}, \theta_{1}, \theta_{2}, q_{1}, q_{2}\right) \\
& :=\frac{1}{2} \int_{0}^{1}\left((\lambda+2 \mu) u_{x}^{2}+\rho u_{t}^{2}+c \theta_{1}^{2}+n \theta_{2}^{2}+\tau_{1} q_{1}^{2}+\tau_{2} q_{2}^{2}+2 d \theta_{1} \theta_{2}\right) d x, \\
E_{2}(t) & :=E_{1}\left(t ; u_{x t}, u_{t t}, \theta_{1 t}, \theta_{2 t}, q_{1 t}, q_{2 t}\right) .
\end{aligned}
$$

The energy $E(t)$ is defined by

$$
E(t):=E_{1}(t)+E_{2}(t)
$$

Our main result reads as follows.

Theorem 2.1 Assume that $u_{0} \in H^{2}(\Omega) \cap H_{0}^{1}(\Omega), u_{1} \in H_{0}^{1}(\Omega), \theta_{1}^{0}, \theta_{2}^{0} \in H^{2}(\Omega) \cap H_{0}^{1}(\Omega)$, $q_{1}^{0}, q_{2}^{0} \in H^{2}(\Omega) \cap H_{*}^{1}(\Omega)$. Then problem (1.1)-(1.7) has a unique global solution such that

$$
\begin{aligned}
& u(t) \in C^{2}\left([0,+\infty), L^{2}(\Omega)\right) \cap C^{1}\left([0,+\infty), H_{0}^{1}(\Omega)\right) \cap C\left([0,+\infty), H^{2}(\Omega) \cap H_{0}^{1}(\Omega)\right), \\
& \theta_{1}(t), \theta_{2}(t) \in C^{1}\left([0,+\infty), L^{2}(\Omega)\right) \cap C\left([0,+\infty), H^{2}(\Omega) \cap H_{0}^{1}(\Omega)\right), \\
& q_{1}(t), q_{2}(t) \in C^{1}\left([0,+\infty), H_{*}^{1}(\Omega)\right) \cap C\left([0,+\infty), H^{2}(\Omega) \cap H_{*}^{1}(\Omega)\right) .
\end{aligned}
$$

Moreover, the associated energy $E(t)$ defined by (2.4) decays exponentially, i.e., there exist positive constants $c_{0}$ and $C_{0}$ such that

$$
E(t) \leq C_{0} e^{-c_{0} t} E(0), \quad \forall t>0 .
$$

Remark 2.1 If the initial value $\left(u_{0}, u_{1}, \theta_{1}^{0}, \theta_{2}^{0}, q_{1}^{0}, q_{2}^{0}\right) \in D\left(A^{n}\right), n \in \mathbb{N}$, $\left(D\left(A^{n}\right)\right.$ will be defined later), then the solution $\left(u, u_{t}, \theta_{1}, \theta_{2}, q_{1}, q_{2}\right) \in C\left([0,+\infty), D\left(A^{n}\right)\right)$, and problem (1.1)(1.7) yields higher regularity in $t$.

\section{Proof of the main result}

We shall divide the proof into two steps: in Step 1, we shall use the semigroup approach to prove the existence of global solutions and the Remark 2.1; Step 2 is devoted to proving the uniform decay of the energy by the boundary control and the multiplier method.

Step 1. Existence of global solutions.

The proof is based on the semigroup approach (see $[4,12])$ that can be used to reduce problem (1.1)-(1.7) to an abstract initial value problem for a first-order evolution equation. In order to choose proper space for (1.1)-(1.7), we shall consider the static system associ- 
ated with them (see [4]). Considering the energy and the property of operator $A$, we can choose the following state space and the domain of operator $A$ for problem (1.1)-(1.7):

$$
\begin{aligned}
& H=H_{0}^{1}(\Omega) \times\left(L^{2}(\Omega)\right)^{3} \times\left(H_{*}^{1}(\Omega)\right)^{2}, \\
& D(A)=\left(H^{2}(\Omega) \cap H_{0}^{1}(\Omega)\right) \times H_{0}^{1}(\Omega) \times\left(H^{2}(\Omega) \cap H_{0}^{1}(\Omega)\right)^{2} \times\left(H^{2}(\Omega) \cap H_{*}^{1}(\Omega)\right)^{2} .
\end{aligned}
$$

Using the same method as in $[4,12]$, we can prove that the operator $A$ generates a $C_{0}$ semigroup of contractions on the Hilbert space $H$. Define

$$
D\left(A^{k}\right)=\left\{y \mid y \in D\left(A^{k-1}\right), A u \in D\left(A^{k-1}\right), k \in \mathbb{N}\right\} .
$$

Then by Theorem 2.3.1 of [13] about the existence and regularities of solutions, we can complete the proof.

Step 2. Uniform decay of the energy.

In this section, we shall assume the existence of solutions in the Sobolev spaces that we need for our computations. The proof of uniform decay is difficult. It is necessary to construct a suitable Lyapunov function and to combine various techniques from energy method, multiplier approaches and boundary control (see $[10,11])$. We mainly refer to Racke [11] for the approaches of thermo-elastic models with second sound.

Multiplying (1.1) by $u_{t},(1.2)$ by $\theta_{1},(1.3)$ by $\theta_{2},(1.4)$ by $q_{1}$, and (1.5) by $q_{2}$ in $L^{2}$, respectively, and summing up the results, yields

$$
\frac{d}{d t} E_{1}(t)=-\int_{0}^{1}\left(q_{1}^{2}+q_{2}^{2}\right) d x
$$

Similarly, we can get

$$
\frac{d}{d t} E_{2}(t)=-\int_{0}^{1}\left(q_{1 t}^{2}+q_{2 t}^{2}\right) d x
$$

Multiplying (1.1) by $\frac{1}{\lambda+2 \mu} u_{x x}$ in $L^{2}$, we get

$$
\begin{aligned}
& \frac{2}{3} \int_{0}^{1} u_{x x}^{2} d x+\frac{\rho}{\lambda+2 \mu} \frac{d}{d t}\left(\int_{0}^{1} u_{t x} u_{x} d x\right) \\
& \leq \frac{\rho \int_{0}^{1} u_{t x}^{2} d x}{\lambda+2 \mu}+\frac{3 \int_{0}^{1}\left(\gamma_{1}^{2} \theta_{1 x}^{2}+\gamma_{2}^{2} \theta_{2 x}^{2}\right) d x}{2(\lambda+2 \mu)^{2}} .
\end{aligned}
$$

Multiplying (1.2) by $\frac{3 \rho}{2(\lambda+2 \mu) \gamma_{1}} u_{t x}$, (1.3) by $\frac{3 \rho}{2(\lambda+2 \mu) \gamma_{2}} u_{t x}$ in $L^{2}$ and summing them up, yields

$$
\begin{aligned}
& \frac{3 \rho}{\lambda+2 \mu} \int_{0}^{1} u_{t x}^{2} d x \\
& \leq \frac{d}{d t} G_{1}(t)+\frac{1}{6} \int_{0}^{1} u_{x x}^{2} d x+h_{1} \int_{0}^{1} \theta_{1 x}^{2} d x+h_{2} \int_{0}^{1} \theta_{2 x}^{2} d x+h_{3} \int_{0}^{1} \theta_{1 x} \theta_{2 x} d x \\
& \quad+\frac{81 \rho^{2} k}{4(\lambda+2 \mu)^{2} \gamma_{1}^{2}} \int_{0}^{1} q_{1 t}^{2} d x+\frac{81 \rho^{2} D}{4(\lambda+2 \mu)^{2} \gamma_{2}^{2}} \int_{0}^{1} q_{2 t}^{2} d x \\
& \quad-\frac{3 \rho \sqrt{k}}{2(\lambda+2 \mu) \gamma_{1}}\left[q_{1} u_{t x}\right]_{x=0}^{x=1}-\frac{3 \rho \sqrt{D}}{2(\lambda+2 \mu) \gamma_{2}}\left[q_{2} u_{t x}\right]_{x=0}^{x=1},
\end{aligned}
$$


where

$$
\begin{aligned}
G_{1}(t):= & \frac{3 \rho \sqrt{k}}{2(\lambda+2 \mu)^{2} \gamma_{1}} \int_{0}^{1}\left(\frac{\rho \sqrt{k}}{\gamma_{1}} q_{1} u_{t t}+\frac{\rho \sqrt{D}}{\gamma_{2}} q_{2} u_{t t}-\tau_{1} q_{1} q_{1 t}-q_{1}^{2}-q_{2}^{2}-\frac{\rho k+\rho D}{\sqrt{k D}} q_{1} q_{2}\right. \\
& \left.-\tau_{2} q_{2} q_{2 t}-\frac{\sqrt{k} \tau_{2}}{\sqrt{D}} q_{1} q_{2 t}-\frac{\sqrt{D} \tau_{1}}{\sqrt{k}} q_{2} q_{1 t}\right) d x-\frac{3 \rho}{2(\lambda+2 \mu) \gamma_{1} \gamma_{2}} \\
& \times \int_{0}^{1}\left[\frac{c \gamma_{2}+d \gamma_{1}}{\sqrt{k}}\left(q_{1} u_{t}+\tau_{1} q_{1 t} u_{t}\right)+\frac{d \gamma_{2}+n \gamma_{1}}{\sqrt{D}}\left(q_{2} u_{t}+\tau_{2} q_{2 t} u_{t}\right)\right] d x \\
h_{3}:= & \frac{3}{\lambda+2 \mu}\left(\frac{c \gamma_{2}}{2 \gamma_{1}}+\frac{n \gamma_{1}}{2 \gamma_{2}}+d\right), \quad h_{1}:=\frac{3}{2}\left(\frac{c}{\lambda+2 \mu}+\frac{d \gamma_{1}}{\gamma_{2}}\right)+\frac{81}{4}\left(\frac{c^{2}}{\gamma_{1}^{2}}+\frac{d^{2}}{\gamma_{2}^{2}}\right), \\
h_{2}:= & \frac{3}{2}\left(\frac{n}{\lambda+2 \mu}+\frac{d \gamma_{2}}{\gamma_{1}}\right)+\frac{81}{4}\left(\frac{d^{2}}{\gamma_{1}^{2}}+\frac{n^{2}}{\gamma_{2}^{2}}\right) .
\end{aligned}
$$

Combining (3.5) with (3.6), we get

$$
\begin{aligned}
& \frac{2 \rho}{\lambda+2 \mu} \int_{0}^{1} u_{t x}^{2} d x+\frac{1}{2} \int_{0}^{1} u_{x x}^{2} d x+\frac{d}{d t}\left(-G_{1}(t)+\frac{\rho}{\lambda+2 \mu} \int_{0}^{1} u_{t x} u_{x} d x\right) \\
& \leq \frac{81 \rho^{2} k}{4(\lambda+2 \mu)^{2} \gamma_{1}^{2}} \int_{0}^{1} q_{1 t}^{2} d x+\left(h_{1}+\frac{h_{3}}{2}+\frac{3 \gamma_{1}^{2}}{2(\lambda+2 \mu)^{2}}\right) \int_{0}^{1} \theta_{1 x}^{2} d x \\
& \quad+\frac{81 \rho^{2} D}{4(\lambda+2 \mu)^{2} \gamma_{2}^{2}} \int_{0}^{1} q_{2 t}^{2} d x+\left(h_{2}+\frac{h_{3}}{2}+\frac{3 \gamma_{2}^{2}}{2(\lambda+2 \mu)^{2}}\right) \int_{0}^{1} \theta_{2 x}^{2} d x \\
& \quad-\frac{3 \rho \sqrt{k}}{2(\lambda+2 \mu) \gamma_{1}}\left[q_{1} u_{t x}\right]_{x=0}^{x=1}-\frac{3 \rho \sqrt{D}}{2(\lambda+2 \mu) \gamma_{2}}\left[q_{2} u_{t x}\right]_{x=0}^{x=1} .
\end{aligned}
$$

Now, we conclude from (1.1), (1.4), (1.5) and Poincaré inequality

$$
\begin{aligned}
\int_{0}^{1}\left(u_{t}^{2}+u_{t t}^{2}+\theta_{1}^{2}+\theta_{2}^{2}\right) d x \\
\leq \frac{2(\lambda+2 \mu)^{2}}{\rho^{2}} \int_{0}^{1} u_{x x}^{2} d x+\frac{2}{D}\left(\frac{2 \gamma_{2}^{2}}{\rho^{2}}+\frac{1}{\pi^{2}}\right) \int_{0}^{1}\left(q_{2}^{2}+\tau_{2}^{2} q_{2 t}^{2}\right) d x \\
\quad+\frac{1}{\pi^{2}} \int_{0}^{1} u_{t x}^{2} d x+\frac{2}{k}\left(\frac{2 \gamma_{1}^{2}}{\rho^{2}}+\frac{1}{\pi^{2}}\right) \int_{0}^{1}\left(q_{1}^{2}+\tau_{1}^{2} q_{1 t}^{2}\right) d x
\end{aligned}
$$

Multiplying (1.1) by $u$ in $L^{2}$, we obtain

$$
\frac{\lambda+2 \mu}{2} \int_{0}^{1} u_{x}^{2} d x \leq \frac{3}{2 \pi^{2}(\lambda+2 \mu)} \int_{0}^{1}\left(\rho^{2} u_{t t}^{2}+\gamma_{1}^{2} \theta_{1 x}^{2}+\gamma_{2}^{2} \theta_{2 x}^{2}\right) d x .
$$

From (1.2) and (1.3), we get

$$
\begin{aligned}
& \theta_{1 t}=-\frac{1}{n c-d^{2}}\left(n \sqrt{k} q_{1 x}-d \sqrt{D} q_{2 x}+\left(n \gamma_{1}-d \gamma_{2}\right) u_{t x}\right), \\
& \theta_{2 t}=-\frac{1}{n c-d^{2}}\left(c \sqrt{D} q_{2 x}-d \sqrt{k} q_{1 x}+\left(c \gamma_{2}-d \gamma_{1}\right) u_{t x}\right) .
\end{aligned}
$$


Multiplying (3.10) by $\theta_{1 t}$, (3.11) by $\theta_{2 t}$ in $L^{2}$, and summing up the results, we get

$$
\begin{aligned}
\int_{0}^{1}\left(\theta_{1 t}^{2}+\theta_{2 t}^{2}\right) d x-\frac{d}{d t} G_{2}(t) \\
\leq \\
\leq \frac{2 k\left(n^{2}+d^{2}\right)}{\left(n c-d^{2}\right)^{2}} \int_{0}^{1} q_{1 t}^{2} d x+\frac{\left(n \gamma_{1}-d \gamma_{2}\right)^{2}+\left(c \gamma_{2}-d \gamma_{1}\right)^{2}}{\left(n c-d^{2}\right)^{2}} \int_{0}^{1} u_{t x}^{2} d x \\
\quad+\int_{0}^{1}\left(\theta_{1 x}^{2}+\theta_{2 x}^{2}\right) d x+\frac{2 D\left(c^{2}+d^{2}\right)}{\left(n c-d^{2}\right)^{2}} \int_{0}^{1} q_{2 t}^{2} d x
\end{aligned}
$$

where $G_{2}(t):=\frac{2}{n c-d^{2}} \int_{0}^{1}\left(n \sqrt{k} q_{1} \theta_{1 x}-d \sqrt{D} q_{2} \theta_{1 x}+c \sqrt{D} q_{2} \theta_{2 x}-d \sqrt{k} q_{1} \theta_{2 x}\right) d x$.

The boundary terms are estimated as follows.

$$
\begin{aligned}
& \left|\left[\frac{3 \rho \sqrt{k}}{2(\lambda+2 \mu) \gamma_{1}} q_{1} u_{t x}\right]_{x=0}^{x=1}\right| \leq \frac{9 \rho^{2} k\left(\left|q_{1}(1)\right|^{2}+\left|q_{1}(0)\right|^{2}\right)}{8(\lambda+2 \mu)^{2} \gamma_{1}^{2} \hat{\varepsilon}}+\frac{\hat{\varepsilon}\left(\left|u_{t x}(1)\right|^{2}+\left|u_{t x}(0)\right|^{2}\right)}{2} \\
& \left|\left[\frac{3 \rho \sqrt{D}}{2(\lambda+2 \mu) \gamma_{2}} q_{2} u_{t x}\right]_{x=0}^{x=1}\right| \leq \frac{9 \rho^{2} D\left(\left|q_{2}(1)\right|^{2}+\left|q_{2}(0)\right|^{2}\right)}{8(\lambda+2 \mu)^{2} \gamma_{2}^{2} \hat{\varepsilon}}+\frac{\hat{\varepsilon}\left(\left|u_{t x}(1)\right|^{2}+\left|u_{t x}(0)\right|^{2}\right)}{2}
\end{aligned}
$$

for some $\hat{\varepsilon}>0$,

$$
\begin{aligned}
& \left|q_{1}(1)\right|^{2}+\left|q_{1}(0)\right|^{2} \leq 2\left(1+\frac{1}{\hat{\varepsilon}^{2}}\right) \int_{0}^{1} q_{1}^{2} d x+\frac{4 \hat{\varepsilon}^{2}}{k} \int_{0}^{1} c^{2} \theta_{1 t}^{2}+d^{2} \theta_{2 t}^{2}+\gamma_{1}^{2} u_{t x}^{2} d x \\
& \left|q_{2}(1)\right|^{2}+\left|q_{2}(0)\right|^{2} \leq 2\left(1+\frac{1}{\hat{\varepsilon}^{2}}\right) \int_{0}^{1} q_{2}^{2} d x+\frac{4 \hat{\varepsilon}^{2}}{D} \int_{0}^{1} d^{2} \theta_{1 t}^{2}+n^{2} \theta_{2 t}^{2}+\gamma_{2}^{2} u_{t x}^{2} d x
\end{aligned}
$$

Combining (3.13)-(3.16), we get

$$
\begin{aligned}
& \left|\left[\frac{3 \rho \sqrt{k}}{2(\lambda+2 \mu) \gamma_{1}} q_{1} u_{t x}\right]_{x=0}^{x=1}\right|+\left|\left[\frac{3 \rho \sqrt{D}}{2(\lambda+2 \mu) \gamma_{2}} q_{2} u_{t x}\right]_{x=0}^{x=1}\right| \\
& \quad \leq \frac{9 \rho^{2}\left(1+\hat{\varepsilon}^{2}\right)}{4(\lambda+2 \mu)^{2} \hat{\varepsilon}^{3}} \int_{0}^{1}\left(\frac{k}{\gamma_{1}^{2}} q_{1}^{2}+\frac{D}{\gamma_{2}^{2}} q_{2}^{2}\right) d x+\hat{\varepsilon}\left(\left|u_{t x}(1)\right|^{2}+\left|u_{t x}(0)\right|^{2}\right) \\
& \quad+\frac{9 \rho^{2} \hat{\varepsilon}}{2(\lambda+2 \mu)^{2}} \int_{0}^{1}\left[\left(\frac{c^{2}}{\gamma_{1}^{2}}+\frac{d^{2}}{\gamma_{2}^{2}}\right) \theta_{1 t}^{2}+\left(\frac{d^{2}}{\gamma_{1}^{2}}+\frac{n^{2}}{\gamma_{2}^{2}}\right) \theta_{2 t}^{2}+2 u_{t x}^{2}\right] d x .
\end{aligned}
$$

Differentiating (1.1) with respect to $t$ and multiplying by $\varphi(x) u_{t x}$, where

$$
\varphi(x):=1-2 x,
$$

we obtain

$$
\begin{aligned}
& \frac{d}{d t}\left(\int_{0}^{1} \rho u_{t t} \varphi u_{t x} d x\right)-\rho \int_{0}^{1} u_{t t}^{2} d x+\frac{(\lambda+2 \mu)}{2}\left(u_{t x}^{2}(1)+u_{t x}^{2}(0)\right)-(\lambda+2 \mu) \int_{0}^{1} u_{t x}^{2} d x \\
& \quad+\gamma_{1} \int_{0}^{1} \theta_{1 t x} \varphi u_{t x} d x+\gamma_{2} \int_{0}^{1} \theta_{2 t x} \varphi u_{t x} d x=0 .
\end{aligned}
$$


Chang and Quin Boundary Value Problems 2013, 2013:222

Page 7 of 11

Multiplications of (1.2) by $\varphi \theta_{1 t x}$ and (1.3) by $\varphi \theta_{2 t x}$ yield

$$
\begin{aligned}
& -c \int_{0}^{1} \theta_{1 t}^{2} d x-\sqrt{k} \frac{d}{d t}\left(\int_{0}^{1} q_{1 x} \varphi \theta_{1 x} d x\right)+\sqrt{k} \int_{0}^{1} q_{1 t x} \varphi \theta_{1 x} d x \\
& =\gamma_{1} \int_{0}^{1} u_{t x} \varphi \theta_{1 t x} d x+d \int_{0}^{1} \theta_{2 t} \varphi \theta_{1 t x} d x, \\
& -n \int_{0}^{1} \theta_{2 t}^{2} d x-\sqrt{D} \frac{d}{d t}\left(\int_{0}^{1} q_{2 x} \varphi \theta_{2 x} d x\right)+\sqrt{D} \int_{0}^{1} q_{2 t x} \varphi \theta_{2 x} d x \\
& =\gamma_{2} \int_{0}^{1} u_{t x} \varphi \theta_{2 t x} d x+d \int_{0}^{1} \theta_{1 t} \varphi \theta_{2 t x} d x,
\end{aligned}
$$

which implies, using (1.4) and (1.5),

$$
\begin{aligned}
-c \int_{0}^{1} \theta_{1 t}^{2} d x-\sqrt{k} \frac{d}{d t}\left(\int_{0}^{1} q_{1 x} \varphi \theta_{1 x} d x\right) \\
-\frac{\sqrt{k}}{\tau_{1}} \int_{0}^{1} q_{1 x} \varphi \theta_{1 x} d x+\frac{k}{2 \tau_{1}}\left(\theta_{1 x}^{2}(1)+\theta_{1 x}^{2}(0)\right) \\
=\frac{k}{\tau_{1}} \int_{0}^{1} \theta_{1 x}^{2} d x+\gamma_{1} \int_{0}^{1} u_{t x} \varphi \theta_{1 t x} d x+d \int_{0}^{1} \theta_{2 t} \varphi \theta_{1 t x} d x \\
-n \int_{0}^{1} \theta_{2 t}^{2} d x-\sqrt{D} \frac{d}{d t}\left(\int_{0}^{1} q_{2 x} \varphi \theta_{2 x} d x\right) \\
-\frac{\sqrt{D}}{\tau_{1}} \int_{0}^{1} q_{2 x} \varphi \theta_{2 x} d x+\frac{D}{2 \tau_{2}}\left(\theta_{2 x}^{2}(1)+\theta_{2 x}^{2}(0)\right) \\
=\frac{D}{\tau_{2}} \int_{0}^{1} \theta_{2 x}^{2} d x+\gamma_{2} \int_{0}^{1} u_{t x} \varphi \theta_{2 t x} d x+d \int_{0}^{1} \theta_{1 t} \varphi \theta_{2 t x} d x=0 .
\end{aligned}
$$

Combining (3.19)-(3.21), we conclude

$$
\begin{aligned}
& \frac{d}{d t}\left(\int_{0}^{1} \rho u_{t t} \varphi u_{t x}-\sqrt{k} q_{1 x} \varphi \theta_{1 x}-\sqrt{D} q_{2 x} \varphi \theta_{2 x} d x\right)+\frac{\lambda+2 \mu}{2}\left(u_{t x}^{2}(1)+u_{t x}^{2}(0)\right) \\
& \leq \rho \int_{0}^{1} u_{t t}^{2} d x+(\lambda+2 \mu) \int_{0}^{1} u_{t x}^{2} d x+c \int_{0}^{1} \theta_{1 t}^{2} d x+n \int_{0}^{1} \theta_{2 t}^{2} d x+\int_{0}^{1} q_{1 x}^{2} d x \\
& \quad+\frac{k}{\tau_{1}} \int_{0}^{1} \theta_{1 x}^{2} d x+\frac{D}{\tau_{2}} \int_{0}^{1} \theta_{2 x}^{2} d x+\frac{k}{4 \tau_{1}^{2}} \int_{0}^{1} \theta_{1 x}^{2} d x \\
& \quad+\frac{D}{4 \tau_{2}^{2}} \int_{0}^{1} \theta_{2 x}^{2} d x+\int_{0}^{1} q_{2 x}^{2} d x .
\end{aligned}
$$

Using (1.2) and (1.3), we get

$$
\begin{aligned}
& \frac{d}{d t}\left(\frac{2 \hat{\varepsilon}}{\lambda+2 \mu} \int_{0}^{1} \rho u_{t t} \varphi u_{t x}-\sqrt{k} q_{1 x} \varphi \theta_{1 x}-\sqrt{D} q_{2 x} \varphi \theta_{2 x} d x\right)+\hat{\varepsilon}\left(u_{t x}^{2}(1)+u_{t x}^{2}(0)\right) \\
& \leq\left(2 \hat{\varepsilon}+\frac{4 \hat{\varepsilon} \gamma_{1}^{2}}{(\lambda+2 \mu) k}+\frac{4 \hat{\varepsilon} \gamma_{2}^{2}}{(\lambda+2 \mu) D}\right) \int_{0}^{1} u_{t x}^{2} d x \\
& \quad+\left(\frac{2 \hat{\varepsilon} c}{\lambda+2 \mu}+\frac{4 \hat{\varepsilon} c^{2}}{(\lambda+2 \mu) k}+\frac{4 \hat{\varepsilon} d^{2}}{(\lambda+2 \mu) D}\right) \int_{0}^{1} \theta_{1 t}^{2} d x
\end{aligned}
$$




$$
\begin{aligned}
& +\frac{2 \hat{\varepsilon} \rho}{\lambda+2 \mu} \int_{0}^{1} u_{t t}^{2} d x+\left(\frac{2 \hat{\varepsilon} n}{\lambda+2 \mu}+\frac{4 \hat{\varepsilon} d^{2}}{(\lambda+2 \mu) k}+\frac{4 \hat{\varepsilon} n^{2}}{(\lambda+2 \mu) D}\right) \int_{0}^{1} \theta_{2 t}^{2} d x \\
& +\left(\frac{2 \hat{\varepsilon} k}{(\lambda+2 \mu) \tau_{1}}+\frac{\hat{\varepsilon} k}{2(\lambda+2 \mu) \tau_{1}^{2}}\right) \int_{0}^{1} \theta_{1 x}^{2} d x \\
& +\left(\frac{2 \hat{\varepsilon} D}{(\lambda+2 \mu) \tau_{2}}+\frac{\hat{\varepsilon} D}{2(\lambda+2 \mu) \tau_{2}^{2}}\right) \int_{0}^{1} \theta_{2 x}^{2} d x
\end{aligned}
$$

With (3.17) and (3.23), we can estimate

$$
\begin{aligned}
& \left|\left[\frac{3 \rho \sqrt{k}}{2(\lambda+2 \mu) \gamma_{1}} q_{1} u_{t x}\right]_{x=0}^{x=1}\right|+\left|\left[\frac{3 \rho \sqrt{D}}{2(\lambda+2 \mu) \gamma_{2}} q_{2} u_{t x}\right]_{x=0}^{x=1}\right| \\
& \leq \frac{9 \rho^{2}\left(1+\hat{\varepsilon}^{2}\right)}{4(\lambda+2 \mu)^{2} \hat{\varepsilon}^{3}} \int_{0}^{1}\left(\frac{k}{\gamma_{1}^{2}} q_{1}^{2}+\frac{D}{\gamma_{2}^{2}} q_{2}^{2}\right) d x+\frac{2 \hat{\varepsilon} \rho}{\lambda+2 \mu} \int_{0}^{1} u_{t t}^{2} d x-\frac{d}{d t} G_{3}(t) \\
& +\hat{\varepsilon}\left(\frac{9 \rho^{2}}{2(\lambda+2 \mu)^{2}}\left(\frac{c^{2}}{\gamma_{1}^{2}}+\frac{d^{2}}{\gamma_{2}^{2}}\right)+\frac{2 c}{\lambda+2 \mu}+\frac{4 c^{2}}{(\lambda+2 \mu) k}+\frac{4 d^{2}}{(\lambda+2 \mu) D}\right) \int_{0}^{1} \theta_{1 t}^{2} d x \\
& +\hat{\varepsilon}\left(\frac{9 \rho^{2}}{2(\lambda+2 \mu)^{2}}\left(\frac{d^{2}}{\gamma_{1}^{2}}+\frac{n^{2}}{\gamma_{2}^{2}}\right)+\frac{2 n}{\lambda+2 \mu}+\frac{4 d^{2}}{(\lambda+2 \mu) k}+\frac{4 n^{2}}{(\lambda+2 \mu) D}\right) \int_{0}^{1} \theta_{2 t}^{2} d x \\
& +\hat{\varepsilon}\left(\frac{2 k}{(\lambda+2 \mu) \tau_{1}}+\frac{k}{2(\lambda+2 \mu) \tau_{1}^{2}}\right) \int_{0}^{1} \theta_{1 x}^{2} d x \\
& +\hat{\varepsilon}\left(\frac{2 D}{(\lambda+2 \mu) \tau_{2}}+\frac{D}{2(\lambda+2 \mu) \tau_{2}^{2}}\right) \int_{0}^{1} \theta_{2 x}^{2} d x \\
& +\hat{\varepsilon}\left(\frac{9 \rho^{2}}{(\lambda+2 \mu)^{2}}+2+\frac{4 \gamma_{1}^{2}}{(\lambda+2 \mu) k}+\frac{4 \gamma_{2}^{2}}{(\lambda+2 \mu) D}\right) \int_{0}^{1} u_{t x}^{2} d x,
\end{aligned}
$$

where $G_{3}:=\frac{2 \hat{\varepsilon}}{\lambda+2 \mu} \int_{0}^{1} \rho u_{t t} \varphi u_{t x}-\sqrt{k} q_{1 x} \varphi \theta_{1 x}-\sqrt{D} q_{2 x} \varphi \theta_{2 x} d x$.

Define $\xi:=\frac{\rho\left(n c-d^{2}\right)^{2}}{4(\lambda+2 \mu)\left[\left(n \gamma_{1}-d \gamma_{2}\right)^{2}+\left(\left(c \gamma_{2}-d \gamma_{1}\right)^{2}\right]\right.}$. Multiplying both sides of (3.12) by $\xi$ and combining the result with (3.7) and (3.24), we obtain for sufficiently small $\hat{\varepsilon}$ the estimate

$$
\begin{aligned}
& \frac{\rho \int_{0}^{1} u_{t x}^{2} d x}{\lambda+2 \mu}+\frac{1}{4} \int_{0}^{1} u_{x x}^{2} d x+\frac{\xi}{2} \int_{0}^{1}\left(\theta_{1 t}^{2}+\theta_{2 t}^{2}\right) d x+\frac{d G(t)}{d t} \\
& \leq C_{1} \int_{0}^{1}\left(q_{1}^{2}+q_{2}^{2}+q_{1 t}^{2}+q_{2 t}^{2}\right) d x
\end{aligned}
$$

where $G(t)=-G_{1}(t)+\frac{\rho}{\lambda+2 \mu} \int_{0}^{1} u_{t x} u_{x} d x-\xi G_{2}(t)+G_{3}(t)$, and $C_{1}=C_{1}\left(\hat{\varepsilon}, \lambda, \mu, \gamma_{1}, \gamma_{2}, k, D, n, c\right.$, $\left.d, \tau_{1}, \tau_{2}\right)$. Now, we can define the desired Lyapunov functional $F(t)$. For $\varepsilon>0$, to be determined later on, let

$$
F(t):=\frac{1}{\varepsilon} E(t)+G(t)
$$

Then we conclude from (3.3), (3.4), and (3.15)

$$
\begin{aligned}
\frac{d}{d t} F(t) \leq & -\frac{1}{\varepsilon} \int_{0}^{1}\left(q_{1}^{2}+q_{2}^{2}+q_{1 t}^{2}+q_{2 t}^{2}\right) d x-\frac{\rho}{\lambda+2 \mu} \int_{0}^{1} u_{t x}^{2} d x-\frac{1}{4} \int_{0}^{1} u_{x x}^{2} d x \\
& -\frac{\xi}{2} \int_{0}^{1}\left(\theta_{1 t}^{2}+\theta_{2 t}^{2}\right) d x+C_{1} \int_{0}^{1}\left(q_{1}^{2}+q_{2}^{2}+q_{1 t}^{2}+q_{2 t}^{2}\right) d x .
\end{aligned}
$$


By using (3.8), we arrive at

$$
\begin{aligned}
& -C_{2} \varepsilon \int_{0}^{1}\left(q_{1}^{2}+q_{2}^{2}+q_{1 t}^{2}+q_{2 t}^{2}\right) d x-\frac{\varepsilon}{\pi^{2}} \int_{0}^{1} u_{t x}^{2} d x-\frac{2 \varepsilon(\lambda+2 \mu)^{2}}{\rho^{2}} \int_{0}^{1} u_{x x}^{2} d x \\
& \quad \leq-\varepsilon \int_{0}^{1}\left(u_{t}^{2}+u_{t t}^{2}+\theta_{1}^{2}+\theta_{2}^{2}\right) d x,
\end{aligned}
$$

while (3.9) yields

$$
-C_{3} \varepsilon^{2} \int_{0}^{1} u_{x x}^{2} d x-C_{4} \varepsilon^{2} \int_{0}^{1}\left(q_{1}^{2}+q_{2}^{2}+q_{1 t}^{2}+q_{2 t}^{2}\right) d x \leq-\varepsilon^{2} \frac{\lambda+2 \mu}{2} \int_{0}^{1} u_{x}^{2} d x
$$

Combining (3.27)-(3.29), we conclude

$$
\begin{aligned}
\frac{d}{d t} F(t) \leq & -\left(\frac{1}{\varepsilon}-C_{1}-C_{2} \varepsilon-C_{4} \varepsilon^{2}\right) \int_{0}^{1}\left(q_{1}^{2}+q_{2}^{2}+q_{1 t}^{2}+q_{2 t}^{2}\right) d x-\varepsilon^{2} \frac{\lambda+2 \mu}{2} \int_{0}^{1} u_{x}^{2} d x \\
& -\left(\frac{2 \rho}{\lambda+2 \mu}-\frac{\varepsilon}{\pi^{2}}\right) \int_{0}^{1} u_{t x}^{2} d x-\left[\frac{1}{4}-\frac{2 \varepsilon(\lambda+2 \mu)^{2}}{\rho^{2}}-C_{3} \varepsilon^{2}\right] \int_{0}^{1} u_{x x}^{2} d x \\
& -\frac{\xi}{2} \int_{0}^{1}\left(\theta_{1 t}^{2}+\theta_{2 t}^{2}\right) d x-\varepsilon \int_{0}^{1}\left(u_{t}^{2}+u_{t t}^{2}+\theta_{1}^{2}+\theta_{2}^{2}\right) d x
\end{aligned}
$$

We choose $0<\varepsilon \leq 1$ such that all terms on the right-hand side of (3.30) become negative,

$$
\text { i.e., } \quad \varepsilon \leq \varepsilon_{1}:=\min \left\{\frac{\rho \pi^{2}}{\lambda+2 \mu}, \frac{\rho^{2}}{32(\lambda+2 \mu)^{2}}, \frac{1}{4} \sqrt{\frac{1}{C_{3}}}, \frac{1}{2\left(C_{1}+C_{2}+C_{4}\right)}\right\} \text {. }
$$

Choosing $\varepsilon$ as in (3.31), we obtain from (3.30)

$$
\frac{d}{d t} F(t) \leq-d_{1} \int_{0}^{1}\left(u_{x}^{2}+u_{t}^{2}+u_{t x}^{2}+u_{t t}^{2}+\theta_{1}^{2}+\theta_{2}^{2}+\theta_{1 t}^{2}+\theta_{2 t}^{2}+q_{1}^{2}+q_{2}^{2}+q_{1 t}^{2}+q_{2 t}^{2}\right) d x
$$

with

$$
d_{1}:=\min \left\{\frac{\varepsilon}{2}, \frac{1}{8}, \frac{\rho}{\lambda+2 \mu}, \frac{(\lambda+2 \mu) \varepsilon^{2}}{4}, \frac{\xi}{4}, \frac{1}{2 \varepsilon}\right\}
$$

which implies

$$
\frac{d}{d t} F(t) \leq-d_{2} E(t), \quad d_{2}:=\frac{d_{1}}{2} \min \left\{\frac{1}{\rho}, \frac{1}{\lambda+2 \mu}, \frac{1}{c}, \frac{1}{n}, \frac{1}{\tau_{1}}, \frac{1}{\tau_{2}}\right\}
$$

There exist positive constants $C_{5}, C_{6}$ and $\varepsilon_{2}$ such that for any $\varepsilon \leq \varepsilon_{2}$ and $t \geq 0$, it holds

$$
C_{5} E(t) \leq F(t) \leq C_{6} E(t)
$$

where $C_{5}, C_{6}$ are determined later on. In fact,

$$
|G(t)| \leq C_{5} E(t)
$$


with $C_{5}:=\max \left\{\frac{2 i_{1}}{\tau_{1}}, \frac{2 i_{2}}{\tau_{2}}, \frac{2 i_{3}}{\tau_{1}}, \frac{2 i_{4}}{\tau_{2}}, \frac{2 i_{5}}{\rho}, \frac{2 i_{6}}{\lambda+2 \mu}, \frac{2 i_{7}}{\rho}, \frac{2 i_{8}}{c}, \frac{2 i_{9}}{n}, \frac{\rho}{(\lambda+2 \mu)^{2}}\right\}$,

$$
\begin{aligned}
& i_{1}:=\frac{3 \rho}{4(\lambda+2 \mu)^{2}}\left[\frac{\rho \sqrt{k}}{\gamma_{1}}+\left(\tau_{1}+2\right)+\frac{k+D}{\sqrt{k D}}+\frac{\sqrt{k} \tau_{2}}{\sqrt{D}}\right]+\frac{3 \rho\left(c \gamma_{2}+d \gamma_{1}\right)}{4(\lambda+2 \mu) \gamma_{1} \gamma_{2} \sqrt{k}} \\
& +\frac{(n+d) \sqrt{k}}{n c-d^{2}}+\left[\frac{n \sqrt{k}+d \sqrt{D}}{n c-d^{2}}+\frac{\hat{\varepsilon} \sqrt{k}}{\lambda+2 \mu}\right] \frac{2}{k}, \\
& i_{2}:=\frac{3 \rho}{4(\lambda+2 \mu)^{2}}\left[\frac{\rho \sqrt{D}}{\gamma_{2}}+\left(\tau_{2}+2\right)+\frac{k+D}{\sqrt{k D}}+\frac{\sqrt{D} \tau_{1}}{\sqrt{k}}\right]+\frac{3 \rho\left(n \gamma_{1}+d \gamma_{2}\right)}{4(\lambda+2 \mu) \gamma_{1} \gamma_{2} \sqrt{D}} \\
& +\frac{(c+d) \sqrt{D}}{n c-d^{2}}+\left[\frac{c \sqrt{D}+d \sqrt{k}}{n c-d^{2}}+\frac{\hat{\varepsilon} \sqrt{D}}{\lambda+2 \mu}\right] \frac{2}{D} \\
& i_{3}:=\frac{3 \rho \tau_{1}}{4(\lambda+2 \mu)^{2}}\left(1+\frac{\sqrt{D}}{\sqrt{k}}\right)+\frac{3 \rho \tau_{1}\left(c \gamma_{2}+d \gamma_{1}\right)}{4(\lambda+2 \mu) \gamma_{1} \gamma_{2} \sqrt{k}}+\left[\frac{n \sqrt{k}+d \sqrt{D}}{n c-d^{2}}+\frac{\hat{\varepsilon} \sqrt{k}}{\lambda+2 \mu}\right] \frac{2 \tau_{1}^{2}}{k}, \\
& i_{4}:=\frac{3 \rho \tau_{2}}{4(\lambda+2 \mu)^{2}}\left(1+\frac{\sqrt{k}}{\sqrt{D}}\right)+\frac{3 \rho \tau_{2}\left(d \gamma_{2}+n \gamma_{1}\right)}{4(\lambda+2 \mu) \gamma_{1} \gamma_{2} \sqrt{D}}+\left[\frac{c \sqrt{D}+d \sqrt{k}}{n c-d^{2}}+\frac{\hat{\varepsilon} \sqrt{D}}{\lambda+2 \mu}\right] \frac{2 \tau_{2}^{2}}{D}, \\
& i_{5}:=\frac{3 \rho\left(1+\tau_{1}\right)\left(d \gamma_{1}+c \gamma_{2}\right)}{4(\lambda+2 \mu) \gamma_{1} \gamma_{2} \sqrt{k}}+\frac{3 \rho\left(1+\tau_{2}\right)\left(d \gamma_{2}+n \gamma_{1}\right)}{4(\lambda+2 \mu) \gamma_{1} \gamma_{2} \sqrt{D}} \\
& i_{6}:=\frac{1}{\lambda+2 \mu}\left[\frac{\rho(1+2 \hat{\varepsilon})}{2}+\frac{2 \hat{\varepsilon} \gamma_{1}^{2}}{\sqrt{k}}+\frac{2 \hat{\varepsilon} \gamma_{2}^{2}}{\sqrt{D}}\right] \text {, } \\
& i_{7}=\frac{\rho}{\lambda+2 \mu}\left[\frac{3 \sqrt{k}}{4(\lambda+2 \mu) \gamma_{1}}+\frac{3 \sqrt{D}}{4(\lambda+2 \mu) \gamma_{2}}+\hat{\varepsilon}\right] \text {, } \\
& i_{8}:=\frac{2 \hat{\varepsilon} c^{2}}{(\lambda+2 \mu) \sqrt{k}}+\frac{2 \hat{\varepsilon} d^{2}}{(\lambda+2 \mu) \sqrt{D}} \text {, } \\
& i_{9}=\frac{2 \hat{\varepsilon} d^{2}}{(\lambda+2 \mu) \sqrt{k}}+\frac{2 \hat{\varepsilon} n^{2}}{(\lambda+2 \mu) \sqrt{D}} \text {. }
\end{aligned}
$$

At this point, we choose $\varepsilon \leq \varepsilon_{2}:=\frac{1}{2 C_{5}}, C_{6}:=\frac{1}{\varepsilon}+C_{5}$. Finally, we choose $\varepsilon:=\min \left\{\varepsilon_{1}, \varepsilon_{2}\right\}$. Thus, we have the validity of (3.34). Combining (3.33) with (3.34), we get

$$
\frac{d}{d t} F(t) \leq-d_{0} F(t), \quad d_{0}:=\frac{d_{2}}{C_{6}} .
$$

Hence, it follows from (3.36), $F(t) \leq e^{-d_{0} t} F(0)$. Applying (3.34) again, we can conclude (2.5) with $C_{0}:=\frac{C_{6}}{C_{5}}$. The proof is complete.

\section{Competing interests}

The authors declare that they have no competing interests.

\section{Authors' contributions}

The paper is a joint work of all authors who contributed equally to the final version of the paper. All authors read and approved the final manuscript.

\section{Author details}

${ }^{1}$ College of Information Science and Technology, Donghua University, Shanghai, 201620, P.R. China. ${ }^{2}$ Department of Applied Mathematics, Donghua University, Shanghai, 201620, P.R. China.

\section{Acknowledgements}

This paper was in part supported by the NNSF of China with contract numbers 11031003, 11271066 and a grant from Shanghai Education Commission $13 Z Z 048$. 


\section{References}

1. Nowacki, W: Certain problem of thermodiffusion. Arch. Mech. 23, 731-754 (1971)

2. Nowacki, W: Dynamical problem of thermodiffusion in solids II. Bull. Pol. Acad. Sci., Tech. Sci. 22, 205-211 (1974)

3. Liu, Y, Reissig, M: Models of thermodiffusion in 1D. Math. Methods Appl. Sci. (2013). doi:10.1002/mma.2839 (in press)

4. Qin, Y, Zhang, M, Feng, B, Li, H: Global existence and asymptotic behavior of solutions for thermodiffusion equations. J. Math. Anal. Appl. 408, 140-153 (2013)

5. Szymaniec, $A: L^{p}-L^{q}$ time decay estimates for the solution of the linear partial differential equations of thermodiffusion. Appl. Math. 37, 143-170 (2010)

6. Podstrigach, Y: Differential equations of the problem of thermodiffusion in an isotropic deformable solid. Dopov Akad. Nauk Ukr. RSR 2, 169-172 (1961)

7. Fichera, G: Uniqueness, existence and estimate of the solution in the dynamical problem of thermodiffusion in an elastic solid. Arch. Ration. Mech. Anal. 26, 903-920 (1974)

8. Gawinecki, JA, Sierpinski, K: Existence, uniqueness and regularity of the solution of the first boundary initial value problem for the equation of the thermodiffusion in a solid body. Bull. Pol. Acad. Sci., Tech. Sci. 30, 541-547 (1982)

9. Szymaniec, A: Global solution to the Cauchy problem of nonlinear thermodiffusion in a solid body. Appl. Math. 37, 437-458 (2010)

10. Jiang, S, Racke, R: Evolution Equations in Thermoelasticity. Monographs Surveys Pure Appl. Math., vol. 112. Chapman \& Hall/CRC, Boca Raton (2000)

11. Racke, R: Thermoelasticity with second sound-exponential stability in linear and non-linear 1-d. Math. Methods Appl. Sci. 25, 409-441 (2002)

12. Liu, Z, Zheng, S: Semigroups Associated with Dissipative Systems. Research Notes in Mathematics, vol. 389. Chapman \& Hall/CRC, Boca Raton (1999)

13. Zheng, S: Nonlinear Evolution Equations. Pitman Monogr. Survey Pure Appl. Math., vol. 133. CRC Press, Boca Raton (2004)

10.1186/1687-2770-2013-222

Cite this article as: Zhang and Qin: Global existence and uniform decay for the one-dimensional model of thermodiffusion with second sound. Boundary Value Problems 2013, 2013:222

\section{Submit your manuscript to a SpringerOpen ${ }^{\odot}$ journal and benefit from:}

- Convenient online submission

- Rigorous peer review

- Immediate publication on acceptance

Open access: articles freely available online

- High visibility within the field

Retaining the copyright to your article 